\title{
Directional Leads for Deep Brain Stimulation: Technical Notes and Experiences
}

\author{
Patrick Fricke $^{\mathrm{a}}$ Robert Nickl $^{\mathrm{a}}$ Maria Breun $^{\mathrm{a}}$ Jens Volkmann ${ }^{\mathrm{b}}$ Dalal Kirsch ${ }^{\mathrm{b}}$ \\ R.-I. Ernestus ${ }^{\mathrm{a}}$ Frank Steigerwald $^{\mathrm{b}}$ Cordula Matthies $^{\mathrm{a}}$

 \\ Würzburg University Hospital, Würzburg, Germany
}

\section{Keywords}

Deep brain stimulation - Directional leads · Functional neurosurgery $\cdot$ Movement disorder surgery $\cdot$ Complications

\begin{abstract}
Objective: Deep brain stimulation (DBS) is an approved treatment for movement disorders. Despite high precision in electrode placement, side effects do occur by current spread to adjacent fibers or nuclei. Directional leads (D-leads) are designed to adapt the volume of stimulation relative to the position within the target by horizontal and vertical current steering directions. The feasibility of implanting these new leads, possible difficulties, and complications were the focus of this study. Material and Methods: This analysis is based on 31 patients who underwent a DBS procedure with Dleads and an implantable pulse generator (IPG) capable of multiple independent current control and 31 patients who received non-D-leads with a similar IPG. While trajectory planning and most steps of the surgical procedure were identical to conventional DBS lead implantation, differences in indication, electrode handling, lead control, and complications were documented and analyzed in comparison to a control group with ring electrodes. Results: During a con-
\end{abstract}

karger@karger.com www.karger.com/sfn

Karger

GOPEN ACCESS
(C) 2021 The Author(s)

Published by S. Karger AG, Basel

This is an Open Access article licensed under the Creative Commons Attribution-NonCommercial-4.0 International License (CC BY-NC) (http://www.karger.com/Services/OpenAccessLicense), applicable to the online version of the article only. Usage and distribution for commercial purposes requires written permission. secutive series of 51 patients implanted with a DBS system, 31 patients $(60.1 \%)$ were selected for implantation of Dleads and received $59 \mathrm{D}$-leads, 28 bilateral, and 3 unilateral implantations. The control group consisted of a consecutive series of a comparable time period, with 31 patients who received conventional ring electrodes. Indication of D-lead implantation was based on the anatomic conditions of the trajectory and target regions and the results of intraoperative test stimulations. In 1 patient, primary D-lead implantation on both sides was performed without any microelectrode implantation to minimize risk for hemorrhage. In the absence of an externally visible marker, the control of implant depth and of the orientation of the D-lead needs to be controlled by X-ray resulting in a longer fluoroscopy time and, therefore, higher $X$-ray dose compared to conventional lead implantations ( 415.53 vs. $328.96 \mathrm{~Gy} \mathrm{~cm}^{2} ; p=0.09$ ). Mean procedure duration for complete system implantation did not differ between either type of leads (ring electrodes vs. Dleads, 08:55 vs. 09:02 h:min). Surgical complications were unrelated to the type of electrode: surgical revision was necessary and successfully performed in 1 subcutaneous hema-

Patrick Fricke, Robert Nickl, Frank Steigerwald, and Cordula Matthies contributed equally. 
toma and 1 unilateral electrode dislocation. A rather rare complication, symptomatic idiopathic delayed-onset edema, was observed in 4 patients with $D$-leads. They recovered completely within 1-3 weeks, spontaneously or after shortterm cortisone medication. In the control group, in a series of 31 patients (20 implanted with Medtronic 3389 lead and 11 with Boston Scientific Vercise lead), not a single problem of this kind was encountered at any time. Conclusion: Precise positioning of D-leads is more challenging than that of conventional DBS leads. By adding an external lead marker, control of optimal lead position and orientation is enhanced. In case of supposed increased risk for hemorrhage because of vessels crossing all possible trajectories in the pre-surgical navigated simulation program, primary D-lead implantation instead of the sharper microelectrodes may be a feasible alternative and it may offer more options than ring electrodes especially in these cases. Prospective studies comparing ring-mode stimulation to directional stimulation to examine the differences of the clinical effects have been started.

$$
\begin{aligned}
& \text { C) } 2021 \text { The Author(s) } \\
& \text { Published by S. Karger AG, Basel }
\end{aligned}
$$

\section{Introduction}

For over 2 decades, deep brain stimulation (DBS) has been shown to be a highly effective treatment for movement disorders, namely, Parkinson's disease, dystonia, and essential tremor $[1,2]$. Still, high precision in electrode placement in the related target areas cannot always prevent side effects from current spread to adjacent fibers or nuclei $[3,4]$. The volume of tissue activated (VTA) has been identified to be of utmost importance for the efficacy of the applied stimulation as well as for the avoidance of side effects [5] and to be related to individual functional anatomy $[6,7]$.

Until 2015, commercially available leads for DBS contained ring electrodes in a linear arrangement, for example, with either 4 (Medtronic Inc., Minneapolis, MN, USA; Abbott (former St. Jude Medical); Little Canada, MN, USA) or 8 electrodes (Boston Scientific, Valencia, CA, USA), allowing limited control of a roughly spherical VTA by polarity, pulse duration, and amplitude settings [5]. Intraoperative experimental studies with so-called Dleads at various setups demonstrated the feasibility of horizontal steering in humans $[8,9]$.

In September 2015, the first D-lead (Cartesia, Boston Scientific) in combination with a multiple independent current source control pulse generator was released in Europe and has been implanted for the first time in our center. The lead is composed of 4 levels of contacts, with an active tip as most distal and a conventional ring electrode as the most proximal. Each of the 2 middle levels consists of 3 "segmented" contacts spanning about $120^{\circ}$ each, whereby the steering of current in the horizontal plane is enabled [10]. First clinical studies showed that effect and adverse effect thresholds were altered in a highly individual manner by horizontal current steering, and the therapeutic window compared to ring-mode DBS was expanded [11-13].

After gathering experience and collecting data prospectively in a consecutive series of $59 \mathrm{D}$-lead implantations in 31 patients from September 2015 to September 2016 at our institution, we highlight the special neurosurgical aspects of directional DBS. The feasibility of applying these new leads in standard DBS procedures and possible early and long-term difficulties and complications were the focus of this study.

\section{Patients, Material, and Methods}

During the period of 1 year, from September 2015 to September 2016 , technical and surgical data were prospectively collected and retrospectively analyzed for all patients implanted with Cartesia electrodes, as a control group served patients implanted with ring electrodes (3389, Medtronic, and Vercise, Boston Scientific) from February 2015 and September 2016.

\section{Target and Lead Planning}

We used image fusion of MRI to stereotactic CT (Leksell, SurgiPlan, Elekta, Stockholm, Sweden), MRI-based individual adjustment of the atlas-based target coordinates, and multi-trajectory microelectrode mapping to guide the implantation identically to our procedure for conventional DBS leads. A decision for implanting a D-lead instead of a conventional lead was made before surgery in 21 cases, based on clinical presentation and patients' preferences and intraoperatively in another 10 cases, if only a single trajectory was feasible for implantation due to anatomic restrictions and test stimulation via the microelectrodes indicated a narrow therapeutic window (small amplitude range before reaching the adverse effect threshold) for the trajectory with the best therapeutic effect.

\section{Lead Handling}

The Cartesia D-lead consists of 8 electrode contacts arranged in 4 levels. The 2 middle ones contain 3 segmented contacts each, which span $120^{\circ}$ and can therefore direct the VTA in a specific direction. An electrode marker above the electrode contacts helps to adjust the orientation by fluoroscopy (Fig. 1). For efficient application of directionality of DBS, it is important to carefully control for implantation depth and to position the 2 segmented electrodes at the best functional target level according to the intraoperative neurophysiological mapping and test stimulation.

Besides the radio-opaque marker above the electrode contacts, the lead does not contain an external, extra-cerebral marker for orientation and handling after insertion. Therefore, some techni- 
Fig. 1. Scheme of D-lead with electrode contacts 2 and 5 oriented in the same direction as the closed part of the integrated marker. Note the slits between the segmented contacts, which become also visible by lateral fluoroscopy (ㄷ Boston Scientific; reprinted with permission).

Fig. 2. The D-lead is marked for its length and its orientation with the external white stopper, by positioning the screw posteriorly towards the open part of the integrated radiopaque marker.
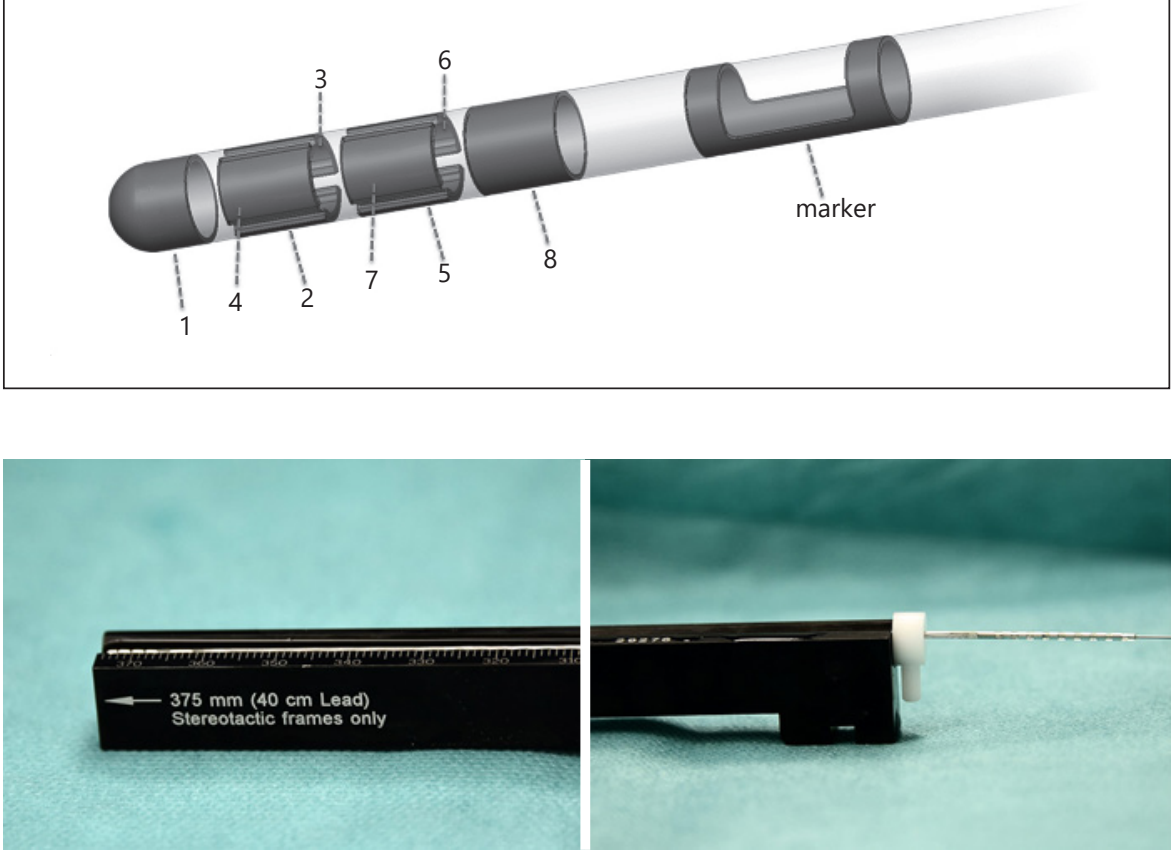

cal support needed to be developed before implantation of the Dlead. The lead is marked externally by the white "lead stopper" for its length and the lead stopper screw is used to indicate the horizontal orientation by aligning it in posterior position, opposite to the closed part of the integrated marker, to indicate the anteriorposterior lead orientation and facilitate its handling (Fig. 2, 3). Furthermore, we mark the lead itself with a permanent sterile marker pen.

\section{Lead Control}

As a standard, we use lateral fluoroscopy through the stereotactic frame to control for implant depth. For D-leads, not only the depth and possible deviation from the planned trajectory are important but also the rotation of the electrode which is controlled by the radiopaque marker above the electrode level. By lateral fluoroscopy, a clear anterior-posterior orientation of the marker and the related electrode contact numbers 2 and 5 in anterior orientation is possible in contrast to a medial or lateral adjustment.

Among the 4 circular poles of the lead, the 2 middle contacts with the directional contacts should be placed at the estimated "sweet spot" chosen for chronic stimulation. After lead placement, while its extra-cerebral part is still secured by the frame, the part of the lead that will be within the bone fixation is coated with a silicone tube. The posterior side of this tube is marked with a permanent pen to facilitate handling the lead rotation during the next steps. After releasing the electrode out of the frame, its depth and rotation are controlled by lateral fluoroscopy (Fig. 4). Correction of the lead orientation should only be performed by clockwise rotation in view of the rotation of the wires within the lead. Lead fixation is carried out with 2 mini titanium plates and 4 screws at the skull (Fig. 5). After securing and fixing the lead, a further test

Experiences with Directional Leads for DBS stimulation in bipolar mode is performed via the upper 2 levels positive and lower 2 levels negative to reproduce effects of the former test stimulation via semi-macroelectrodes to confirm effect and side effect thresholds.

\section{Postoperative Surgical Control}

A postoperative cranial CT scan with image fusion to the presurgically fused set of MRI and stereotactic CT were performed to assess possible surgical sequels as well as the electrode position.

\section{Clinical Programming}

A monopolar review was performed on days 5-8 after surgery as soon as testable off-period symptoms returned, at first by identification of response and side effects level and related electrode level, thereafter by evaluating the individual therapeutic window at contacts with best response.

\section{Follow-Up}

At 2-3 months after implantation, patients were readmitted to the neurology department for an extensive monopolar review and fine tuning of DBS settings. At the same time, a control CT or rotational fluoroscopy was performed for final determination of lead tip location and orientation in relation to preoperative planning.

\section{Results}

From September 2015 to September 2016, out of a consecutive series of 51 patients undergoing a DBS procedure, 31 patients $(60.8 \%$; 8 female, median age 60.5 years, 


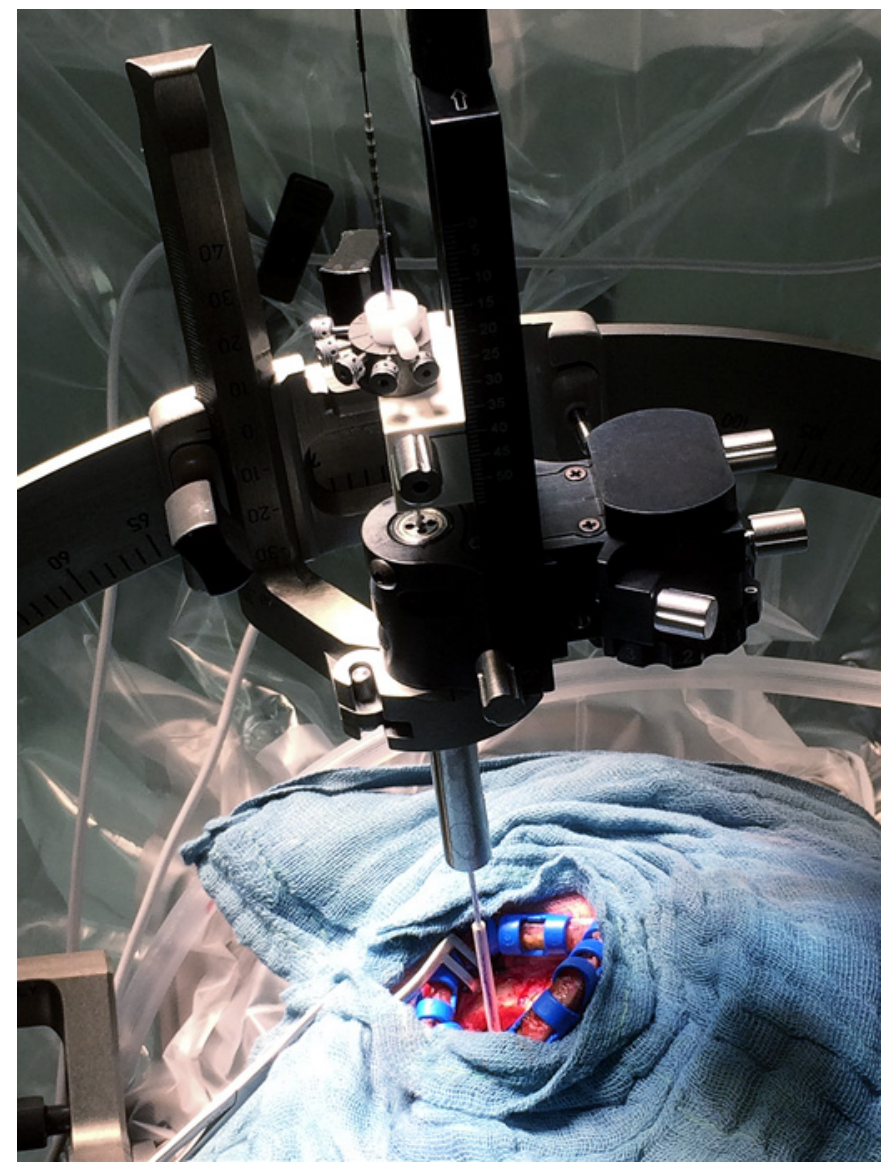

Fig. 3. The screw of the white stopper indicates the horizontal orientation of the directional segments and helps controlling the lead orientation.

range 16-79 years) were implanted with 59 segmented D-leads (Cartesia, Boston Scientific, Valencia, CA, USA), 3 patients received unilateral and 28 bilateral implantations. DBS indications were Parkinson's disease in 27 cases with STN as target, 2 patients had essential tremor and electrodes were implanted in the nucleus ventralis intermedius thalami and retro-subthalamic field, and 2 patients with dystonia had implantations in the globus pallidus internus (GPi).

Implantation of D-lead was offered to every DBS patient since September 2015 except patients with the need for any postoperative MRI, until MRI-approved pulse generators were available. There were 4 cases of revision surgery of previously implanted conventional electrodes that were replaced by $\mathrm{D}$-leads.

The control group consisted also of 31 patients (11 female, median age 58.1 years, range $8-80$ years), 20 of the same time period as the D-lead patients and 11 immedi-
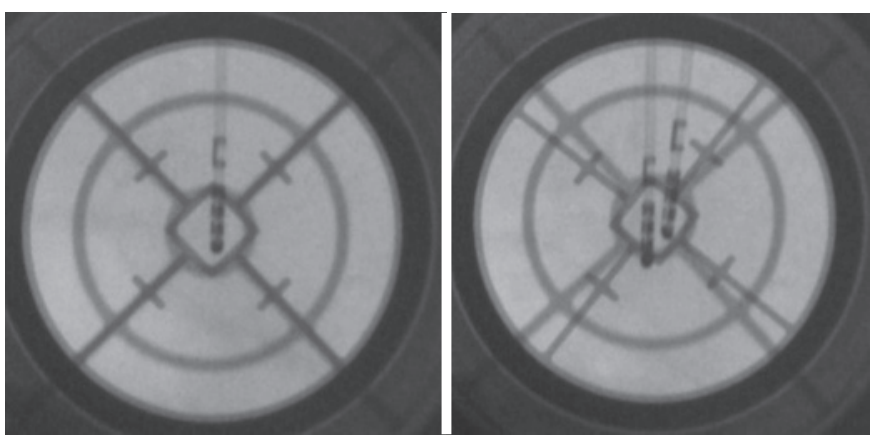

Fig. 4. Directional leads (Cartesia by Boston Scientific) by intraoperative fluoroscopy by left lateral view. The lead marker is oriented anteriorly, the iron sights between anterior and posterolateral respectively between anterior and posteromedial (dotted arrow) directional contacts are clearly visible.

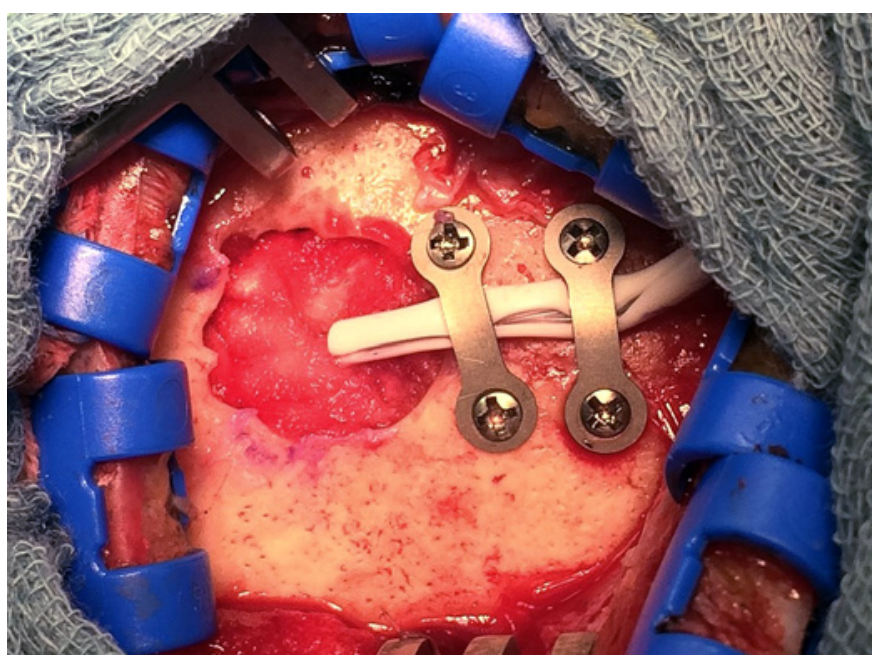

Fig. 5. After coating the lead with a silicone tube, it is fixed in the bony groove by 2 mini titanium plates.

ately beforehand. They received conventional ring electrodes, 20 with Medtronic 3389 and 11 with Boston Scientific Vercise leads.

We did not observe intracranial hematomas, cerebral infarctions, or infections in either patient group. Two surgical complications required surgical revisions in the D-lead group. A subcutaneous hematoma at the impulse generator site was evacuated on the implant day.

Suboptimal electrode position was identified in $1 \mathrm{pa}-$ tient unilaterally ( 1 of 59 ) by postoperative CT imaging; as it occurred on the clinically less affected side, the patient showed bilateral improvement with stimulation, but 
had bothersome residual symptoms contralateral to the misplaced lead despite several programming attempts. After discussing the findings and the possible long-term importance, he agreed on revision which was carried out successfully.

Idiopathic delayed-onset edema was encountered solely in the D-lead group. Four patients became symptomatic after 1-2 uneventful weeks after surgery, by prolonged drowsiness in 3 cases and by leg paresis and apathy in 1 case. Cranial CT identified unilateral edema along the electrode in 3 cases and bilateral edema in 1 patient. All 4 patients recovered completely within a maximum of 3 weeks spontaneously and in 2 patients after IV/oral cortisone treatment.

\section{Surgical Methods and Technique}

The specific technique of marking the D-lead for length and orientation with the external white stopper as well as the control by comparing the stopper to the fluoroscopy findings was applied as reported above and could be fulfilled in each D-lead implantation in all the patients. In each case, care was taken to achieve an anterior-posterior orientation with contacts 2 and 5 in an anterior position and with the slits between the anterior and the posterolateral or posteromedial contacts appearing at the posterior third in the lateral view.

\section{Intraoperative Fluoroscopy}

A slightly longer fluoroscopy time (2.29 min vs. 1.63 $\mathrm{min}$ ) and therefore higher X-ray dose was needed for the placement of the $\mathrm{D}$-leads compared to conventional leads (415.53 vs. $328.96 \mathrm{~Gy} \mathrm{~cm}^{2} ; p=0.09$ ), but the difference was not significant.

\section{Duration of Surgery}

The mean duration of the whole procedure from placement of the stereotactic frame until finishing the impulse generator implantation did not differ between D-lead and conventional lead implantation (09:02 vs. 08:55 h:min). All the patients showed good overall recovery and satisfying response to DBS treatment. At a minimum follow-up of at least 2 months after the implantation, 47 (79.7\%) leads stimulated in steering current mode and 12 (20.3\%) leads in ring mode. With the implementation of current steering leads and the introduction of image-based programming in our institution, the modeling of the approximated optimum VTA was more likely to be obtained by a horizontal steering option. The clinical benefit of this is still under research.

Experiences with Directional Leads for DBS

\section{Change of Stereotactic Procedure with D-Lead}

In 1 patient, at stereotactic planning, multiple vessels were crossing the trajectory and also all simulated alternative trajectories on one side in this patient. By abdication of microelectrodes, direct primary D-lead implantation was performed on the specific side in order to minimize the risk of hemorrhage and to compensate for the lack of alternative trajectories.

\section{Discussion}

\section{Complications and Risks}

In this early series with D-leads, their application compared to ring leads did not expose to greater risks such as cerebral hemorrhage, infection, or electrode deviation. Electrode insertion was as smooth as with all the other available leads and did not cause any direct additional lesions.

But 4 patients (12.9\%) developed idiopathic delayedonset edema around the course of 1 or both implanted electrodes. The patients recovered completely after treatment with cortisone or spontaneously. In the control group, none out of 31 patients (20 with Medtronic 3389 and 11 with Boston Scientific Vercise) revealed any brain edema. Furthermore, before the introduction of D-leads in our institution, during the previous series of 142 consecutive DBS procedures implanted with ring leads over 5 years, there were only 2 unilateral cases of idiopathic delayed-onset edema ( $1.4 \%$ of the patients or $0.7 \%$ of the implanted ring leads). This corresponds to the very low incidence reported about after conventional lead implantations $[12,14]$.

Hence, the increased edema incidence, in 5/59 (8.5\%) implanted D-leads ( 1 bilateral and 3 unilateral cases), is a matter of concern. At present, the underlying cause is completely unknown. Two aspects must be taken into consideration: Though D-leads appear very soft and smooth at the surface, some cause of irritation cannot be excluded coming from the slit sites. In addition, these electrodes were rotated while lying in the brain tissue, what may cause additional irritation. In many cases, a postoperative rotation of the leads was observed, so that the mechanical stress to the brain tissue might last for a prolonged time. Furthermore, these leads are produced in a handmade fashion; whether this might lead to accumulation of any artificial deposits or to significant electrostatic surface changes is a matter of speculation. As to date, the phenomenon of idiopathic delayed-onset edema is neither well understood nor explained; the higher inci- 
dence in $\mathrm{D}$-leads compared to ring leads remains open to speculation [15]. Fortunately, no neurological long-term sequelae were observed in any affected patient.

Electrode misplacement may occur in any stereotactic procedure, and at an increased risk especially in advanced brain atrophy. Reported incidences in the literature vary enormously from $5 \%$ to over $30-40 \%$ [16-18]. The encountered unilateral misplacement in 1 out of $59 \mathrm{im}$ planted leads in this series was small $(\sim 3.0 \mathrm{~mm}$ medial) and most likely due to a combination of brain shift on the second surgical side in brain atrophy and limited and rather misleading cooperation by the patient. Therefore, the revision in this patient was carried out in general anesthesia. This lead dislocation and the indication for revision were not attributable to the type of selected electrode.

In case of increased risk for hemorrhage because of the individual anatomy with multiple crossing vessels, primary D-lead implantation instead of the more pointed microelectrodes may be a feasible alternative. In contrast to conventional electrodes, if the position of the lead cannot be changed, D-leads provide more options to adapt the electric field. Still it is not yet known which deviation of the electrode to the planned target point could be compensated for by $\mathrm{D}$-leads.

\section{Surgical Handling}

For optimum outcome with D-leads, surgical handling needs special attention to adjust implantation depth, with the segmented levels aimed at the best stimulation depth and an adapted technique to control for horizontal lead orientation.

Control of lead orientation, at present, is possible by radiological control of the integrated marker above the active contacts, to prove anterior-posterior orientation. However, the product does not contain any markers that will be visible after insertion of the marker and the active leads into the brain.

The lead does not only tend to shift in a vertical direction, upward or downward dislocation, but also tend to rotate horizontally. For minimizing fluoroscopy control shots, an external marker above the brain surface is helpful during and after the lead insertion. This marker at first may consist of the screw at the lead stopper and second of a slit silicone layer or ink mark.

Correction of lead orientation, once inserted by $60-70$ $\mathrm{mm}$ at depth from the surface, may be achieved by clockwise rotation (in the direction of the turn of the isolated lead wires within the lead carrier) to the optimal position, guided by the external marker and the fluoroscopic control of the radiopaque marker. The externally applied silicone tube serves as a protection at the bony edge and towards the titanium plates. Furthermore, it may also serve as an external marker. As is known from postsurgical investigations [19], D-lead orientation may still change to some degree within the brain, but the primary positioning at surgery poses the important base for the individual long-term condition.

If multiple vessel formation or other anatomical variants put the implantation of multiple microelectrodes at a very high-risk level, primary D-lead implantation may be a good compromise and alternative. Instead of implantation of 1 central microelectrode and the possible shift after its removal, the direct application of D-lead allows good functional intraoperative testing at various directions. With only 1 inserted electrode, it is not possible to choose the best trajectory for final implantation out of the intraoperative microelectrode tests. In this case, D-leads might provide a broader field for adjustment of the VTA than conventional leads.

\section{Optimal Lead Orientation}

Instead of the AP orientation of D-lead presented here, an implantation in medial-lateral orientation of the marker and related active segments is another option favored by some colleagues. This might make the fluoroscopic identification more difficult because the displayed marker could point medial or lateral. Knowing the orientation of the D-lead makes it much easier to program an effective VTA and saves time of testing. Therefore, based on the experience in this case series, AP orientation of Dlead appears as the technique of choice for easy and precise control of the electrode position.

\section{New Lead Models}

When comparing the advantages of the D-lead to the other recent lead model with 8 ring contacts, D-lead seems to bear more advantages for the majority of cases. In our experience, there are few indications where 8 electrode contacts over a length of $15.5 \mathrm{~mm}$ (in contrast to 7.5 $\mathrm{mm}$ in D-lead) will be necessary.

From a surgeon's point of view, the precise implantation of the D-leads is more challenging, but well feasible as demonstrated here. The increase in X-ray dose is small and appears acceptable. The first impressions of the clinical effects indicate that the neurosurgical burden is paid off by a larger therapeutic window [11] and reductions in the therapeutic current amplitude [12]. In this respect, Dleads might compensate small deviations of the lead from an optimal functional target. Prospective studies to compare ring-mode stimulation to directional stimulation are needed and are already under way. 


\section{Summary and Conclusions}

In summary, this feasibility investigation for D-leads proves their reliable and safe clinical applicability and, as well, the necessity for specific technical care at surgical application. Optimal placement of the D-lead is of utmost importance before a realistic comparison of the clinical effects by D-leads versus ring leads can be accomplished. The implantation of the segmented contacts needs to be exactly aligned in depth with the functional target and not every dislocation can be balanced by the adjusted electric field of the segmented contacts. The rotation of the lead is an additional surgical step, which needs exact alignment under fluoroscopy. In cases, in which by reason of crossing vessels only 1 trajectory to the target point is possible, D-leads can compensate for the less favorable anatomical circumstances and minimize the surgical risk.

If adequately placed at stereotactic implantation, Dleads provide many options to shape the activated electric field, if the programming neurologist invests sufficient time for testing and adjusting the stimulation parameters. Ongoing prospective studies evaluate the outcome of Dleads with different time patterns of upcoming positive or negative effects compared to ring-mode stimulation.

\section{Statement of Ethics}

All patients gave their written informed consent to the procedure, and the study was approved by the local Ethics Committee.

\section{Conflict of Interest Statement}

Dr. Fricke reports no conflicts of interest. Dr. Nickl has received grant support from Medtronic and Boston Scientific and honoraria for speaking from Boston Scientific, outside the submitted work. Dr. Breun reports no conflict of interest. Dr. Volkmann reports grants and personal fees from Medtronic Inc., grants and personal fees from Boston Scientific, and personal fees from St. Jude, outside the submitted work. Dr. Kirsch reports no conflicts of interest. Dr. Ernestus reports no conflicts of interest. Dr. Steigerwald has received grant support from Medtronic and Boston Scientific and honoraria for speaking from Boston Scientific, outside the submitted work. Dr. Matthies reports congress fees and honoraria for presentations from Boston Scientific, outside the submitted work.

\section{Funding Sources}

This study did not receive any funding.

\section{Author Contributions}

Each author has made substantial contributions to the conception or design of the work or to the acquisition, analysis, or interpretation of data for the work; participated in drafting the work or revising it critically for important intellectual content; approved the final version to be published; and agreed to be accountable for all aspects of the work in ensuring that questions related to the accuracy or integrity of any part of the work are appropriately investigated and resolved literature.

\section{References}

1 Deuschl G, Schade-Brittinger C, Krack P, Volkmann J, Schäfer H, Bötzel K, et al. A randomized trial of deep-brain stimulation for Parkinson's disease. N Engl J Med. 2006; 355(9):896-908.

2 Benabid AL, Pollak P, Gervason C, Hoffmann D, Gao DM, Hommel M. Long-term suppression of tremor by chronic stimulation of the ventral intermediate thalamic nucleus. Lancet. 1991 Feb 16;337(8738):403-6.

3 Maks CB, Butson CR, Walter BL, Vitek JL, McIntyre CC. Deep brain stimulation activation volumes and their association with neurophysiological mapping and therapeutic outcomes. J Neurol Neurosurg Psychiatry. 2009;80(6):659-66.

4 Wodarg F, Herzog J, Reese R, Falk D, Pinsker MO, Steigerwald F, et al. Stimulation site within the MRI-defined STN predicts postoperative motor outcome. Mov Disord. 2012 Jun;27(7):874-9.
5 Volkmann J, Moro E, Pahwa R. Basic algorithms for the programming of deep brain stimulation in Parkinson's disease. Mov Disord. 2006 Jun;21(Suppl 14):S284-9.

6 Astrom M, Diczfalusy E, Martens H, Wardell K. Relationship between neural activation and electric field distribution during deep brain stimulation. IEEE Trans Biomed Eng. 2015 Feb;62(2):664-72.

7 Reich MM, Brumberg J, Pozzi NG, Marotta G, Roothans J, Åström M, et al. Progressive gait ataxia following deep brain stimulation for essential tremor: adverse effect or lack of efficacy? Brain. 2016;139(11):2948-56.

8 Contarino MF, Bour L, Verhagen R. Directional steering: a novel approach to deep brain stimulation. Neurology. 2014 Sep 23;83(13): 1163-9.

9 Claudio Pollo C, Kaelin-Lang A, Schüpbach M. Directional deep brain stimulation: an intraoperative double-blind pilot study. Brain. 2014;137(7):2015-26.
10 Schüpbach WMM, Chabardes S, Matthies C, Pollo C, Steigerwald F, Timmermann L, et al. Directional leads for deep brain stimulation: opportunities and challenges. Mov Disord. 2017 Oct;32(10):1371-5.

11 Steigerwald F, Müller L, Johannes S, Matthies C, Volkmann J. Directional deep brain stimulation of the subthalamic nucleus: a pilot study using a novel neurostimulation device. Mov Disord. 2016;31(8):1240-3.

12 Rebelo P, Green AL, Aziz TZ, Kent A, Schafer $\mathrm{D}$, Venkatesan L, et al . Thalamic directional deep brain stimulation for tremor: spend less, get more. Brain Stimul. 2018 May-Jun;11(3): 600-6.

13 Dembek TA, Reker P, Visser-Vandewalle V, Wirths J, Treuer H, Klehr M, et al. Directional DBS increases side-effect thresholds: a prospective, double-blind trial. Mov Disord. 2017 Oct;32(10):1380-8.

14 Deogaonkar M, Nazzaro J, Machado JM, Rezai A. Transient, symptomatic, post-operative, non-infectious hypodensity around the deep brain stimulation (DBS) electrode. J Clin Neurosci. 2011 Jul;18(7):910-5. 
15 de Cuba CM, Albanese A, Antonini A, Cossu G, Deuschl G, Eleopra R, et al. Idiopathic delayed-onset edema surrounding deep brain stimulation leads: insights from a case series and systematic literature review. Parkinsonism Relat Disord. 2016 Nov;32:108-15.

16 Okun MS, Tagliati M, Pourfar M, Fernandez $\mathrm{HH}$, Rodriguez RL, Alterman RL, et al. Management of referred deep brain stimulation failures: a retrospective analysis from 2 movement disorders centers. Arch Neurol. 2005; 62(8):1250-5.

17 Rolston JD, Englot DJ, Starr PA, Larson PS An unexpectedly high rate of revisions and removals in deep brain stimulation surgery: analysis of multiple databases. Parkinsonism \& related disorders. Parkinsonism Relat Disord. 2016 Dec;33:72-7.
18 Falowski SM, Bakay RA. Revision surgery of deep brain stimulation leads. Neuromodulation. 2016 Jul;19(5):443-50.

19 Treuer H, Hellerbach A, Borggrefe J, VisserVandewalle V. Regarding "determining the orientation of directional deep brain stimulation electrodes using 3D rotational fluoroscopy". AJNR Am J Neuroradiol. 2017 Dec; 38(12):E105 